\title{
Comparison of Potential Nerve Scar Agents in the Rat Model
}

\author{
David Mossad, MD ${ }^{1}$ Sapan Shah, BS ${ }^{2}$ Farid Amirouche, PhD ${ }^{1}$ Giovanni F. Solitro, PhD ${ }^{1}$ \\ Cory Helder, BS ${ }^{1}$ Alfonso Mejia, MD, MPH ${ }^{1}$ Mark H. Gonzalez, MD, PhD ${ }^{1}$ James M. Kerns, PhD ${ }^{1}$ \\ ${ }^{1}$ Department of Orthopaedics, University of Illinois at Chicago, \\ College of Medicine, Chicago, Illinois \\ 2 University of Illinois at Chicago, College of Medicine, \\ Chicago, Illinois \\ Address for correspondence James M. Kerns, PhD, Department of \\ Orthopaedics, University of Illinois at Chicago, $835 \mathrm{~S}$. Wolcott Avenue, \\ E270, Chicago, IL 60612 (e-mail: Jkerns.kerns@gmail.com).
}

J Reconstr Microsurg Open 2016;1:82-87.

\begin{abstract}
Keywords

- nerve

- rat model

- scar

Background An animal nerve scar model that can be used to identify potential therapies in the treatment of nerve scarring in patients has yet to be defined. The purpose of this study was to create an inexpensive, reproducible, and reliable nerve scar model in the rat.

Methods Thirty rats underwent left tibial nerve transection and repair and were divided into three groups: 10 controls with transection and repair alone, 10 receiving topical application of talc powder onto the repair, and 10 receiving topical application of tetracycline onto the repair. Outcome measures included weekly extensor postural thrust (EPT) testing for 12 weeks, endpoint gastrocnemius weight ratios (left vs right), and histological analysis.

Results The tetracycline group showed a significant difference in EPT testing at week 6 postoperatively ( $p=0.007$ ) and had the lowest average EPT value at each time point over 12 weeks. Each group displayed a plateau in recovery, with the control and talc groups reaching plateau 1 week earlier (week 6) than the tetracycline group (week 7). At 12 weeks postoperatively, there was no significant difference in EPT testing $(p=0.301)$ or gastrocnemius weight ratios $(p=0.802)$. Histological analysis showed substantial nerve regeneration in the control group, an inflammatory response in the epineurium of the talc and tetracycline groups, but no migration of inflammation or interference of nerve regeneration in the endoneurial compartment.

Conclusion These agents, and more importantly, this animal model are inadequate for developing a nerve scar. Identifying a better animal with more connective tissue should be further explored.
\end{abstract}

In the setting of nerve injury, crush injuries maintain epineural and perineural integrity which facilitates recovery while transection injuries introduce a defect which hinders the degree of functional recovery. In addition, transection injuries show an increased amount of collagen or scar formation both around the site of injury and intraneurally distal to the site of injury. ${ }^{1}$

received

September 11, 2015

accepted after revision

May 26, 2016

published online

July 1, 2016
DOI http://dx.doi.org/ 10.1055/s-0036-1584936. ISSN 2377-0813.
Clinically, extraneural scarring surrounding the transection causes neural tissue to adhere to adjacent tissues, compress the nerve, and compromise the blood supply. These tissue alterations can ultimately augment the nerve defect or limit healing potential. $^{2}$ Surgical, pharmacological, and radiative therapies have been attempted to address the defect and scar. ${ }^{3-6}$ Surgical techniques, for example, include microsurgical suture

Copyright $\odot 2016$ by Thieme Medical Publishers, Inc., 333 Seventh Avenue, New York, NY 10001, USA. Tel: +1(212) 584-4662.

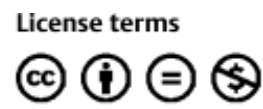

(c) $(1) \triangleq \$$ 
reapproximation repair at either the level of the epineurium or perineurium to shift the site of repair proximal to the site of the traversing axons, or utilizing a graft to create a tension-free repair. $^{3}$ Pharmacological therapies to increase regeneration typically target limiting the inflammatory response. ${ }^{7}$

The formation of a scar and/or neuroma at the injury site along with the subsequent recovery of nerve function may be similar in rats and humans. However, rats may benefit from a more robust recovery. ${ }^{8}$ Paramount in translational research is having a cost-effective, reproducible, and similar model as human nerves in which to test novel therapies. In our study, we sought to use chemical sclerosing agents, talc and tetracycline, ${ }^{9}$ in a standard rat sciatic nerve transection model to induce a better approximation of the lesion found in human transection injuries. ${ }^{10}$ Currently, there is sparse data published regarding whether these interventions affected nerve regeneration and downstream muscle malfunction. ${ }^{11}$ If this could be demonstrated in the rat model, a variety of potential treatments to limit the scar effect could be tested and translated to clinical situations.

\section{Materials and Methods}

\section{Experimental Animals and Surgery}

This study used a total of 30 allogeneic rats (Charles River Fischer $344 ; 200-250 \mathrm{~g}$ ). After acclimation to the animal quarters, all rats received a left-sided tibial nerve transection and microsurgical repair. They were separated into three groups based on the scar-inducing intervention: control group (saline rinse only; $N=10$ ), tetracycline group $(N=10)$, and talc group $(N=10)$. All experimental procedures were conducted under the National Institute of Health Guide for the Care and Use of Laboratory Animals and the Institutional Animal Care and Use Committee at the University of Illinois at Chicago.

The preoperative procedure began with an intraperitoneal injection of ketamine $(90 \mathrm{mg} / \mathrm{kg}$ ) and xylazine $(7.5 \mathrm{mg} / \mathrm{kg})$ for surgical anesthesia, determined by a toe-pinch. After shaving the left lateral thigh, $10 \%$ povidone-iodine solution was applied. Using microsurgical aseptic technique, the left sciatic nerve and its branches (tibial, peroneal, and sural) were identified with a lateral approach and blunt dissection through the biceps femoris muscle. All rats had their left tibial nerve transected midthigh $\sim 3 \mathrm{~mm}$ distal to the trifurcation. The control group consisted of transection and repair alone with 2 to 3 microsutures (9-0 Ethilon), followed by a saline rinse. The tetracycline group had an identical surgery, but with an additional step of flooding the transection site with tetracycline to induce scar formation. The third group had a slurry of talc powder mixed with sterile saline and administered upon the nerve lesion site to induce scar formation.

\section{Extensor Postural Thrust}

This functional outcome measure quantifies the extensor postural thrust (EPT) movement, measured preoperatively and at weekly postoperatively intervals for 12 weeks after a plateau had been attained. The rat was held above the platform of a digital scale, exposing one limb at a time to the scale, and measuring the force in grams upon contact (the heel does not contact the platform). At least 10 trials were conducted for each hind limb, and the mean values were used to calculate (left divided by right) a percent motor recovery (PMR). ${ }^{12}$ The testing was performed primarily by one designated person (S. S.).

\section{Gastrocnemius Muscle Ratios}

This is a gross outcome that compares the wet weights of the left experimental gastrocnemius muscle to the right unoperated gastrocnemius muscle as a ratio. Harvest of the muscles was done at 12 weeks after the final EPT testing to determine the extent of denervation atrophy.

\section{Scar Histology}

This outcome examined the scar formed around the nerve using histology. Harvest of the tibial nerve was done just prior to the muscle harvest. The nerves from both sides were dissected as $10 \mathrm{~mm}$ segments to include the proximal level of the lesion and repair site. Tissues were fixed by immersion in Karnovsky's aldehyde fixative in phosphate buffer for 3 days, rinsed in buffer $(0.1 \mathrm{M})$, and processed for paraffin embedding. Sections were collected at $1 \mathrm{~mm}$ intervals proximally to the suture site. Staining was by the Masson's trichrome stain for connective tissue (blue).

\section{Results}

-Fig. 1 shows a graphical representation of the PMR for the three groups. The recovery was best approximated with a model with two time periods: recovery and plateau. During the first phase for the control group, in the first 6 weeks, PMR increases more rapidly from a low of $24 \%$ to reach a plateau of $65 \%$. The second phase was best represented by a plateau with marginal gains from week 7 to 12, reaching a peak value of $75 \%$. The recovery pattern for the talc group is similar to the control group. The tetracycline group has a slower recovery overall, and significantly less at the 6-week time-point. The Friedman test shows that a significant difference occurs only at the 6 -week time-point ( $p=0.0074)$. With the KruskalWallis test, the means for the talc and control groups are not different $(p=0.94)$ but the talc and tetracycline group are significantly different $(p=0.0006)$. - Fig. 1D shows the overall comparison.

At the time of sacrifice, the surgical site was exposed and photographed to show the gross features of the respective scars (-Fig. 2). The field was dense with white material in the talc group and appeared yellow in the tetracycline group (-Fig. 2B and 2C, respectively). In all cases, the appearance of the gastrocnemius muscles on the experimental left side when compared with the unoperated right side indicated muscle atrophy (-Fig. 3). At 12 weeks, the average left versus right gastrocnemius wet weight ratio was nearly identical for the three groups (control group, $0.61 \pm 0.09$; talc group, $0.61 \pm 0.06$; tetracycline group. $0.58 \pm 0.13$ ). Analysis of variance showed no statistically significant difference between the three groups $(p=0.802)$. The muscle ratios did not correlate with the EPT values $\left(r^{2}=0.054\right)$. 

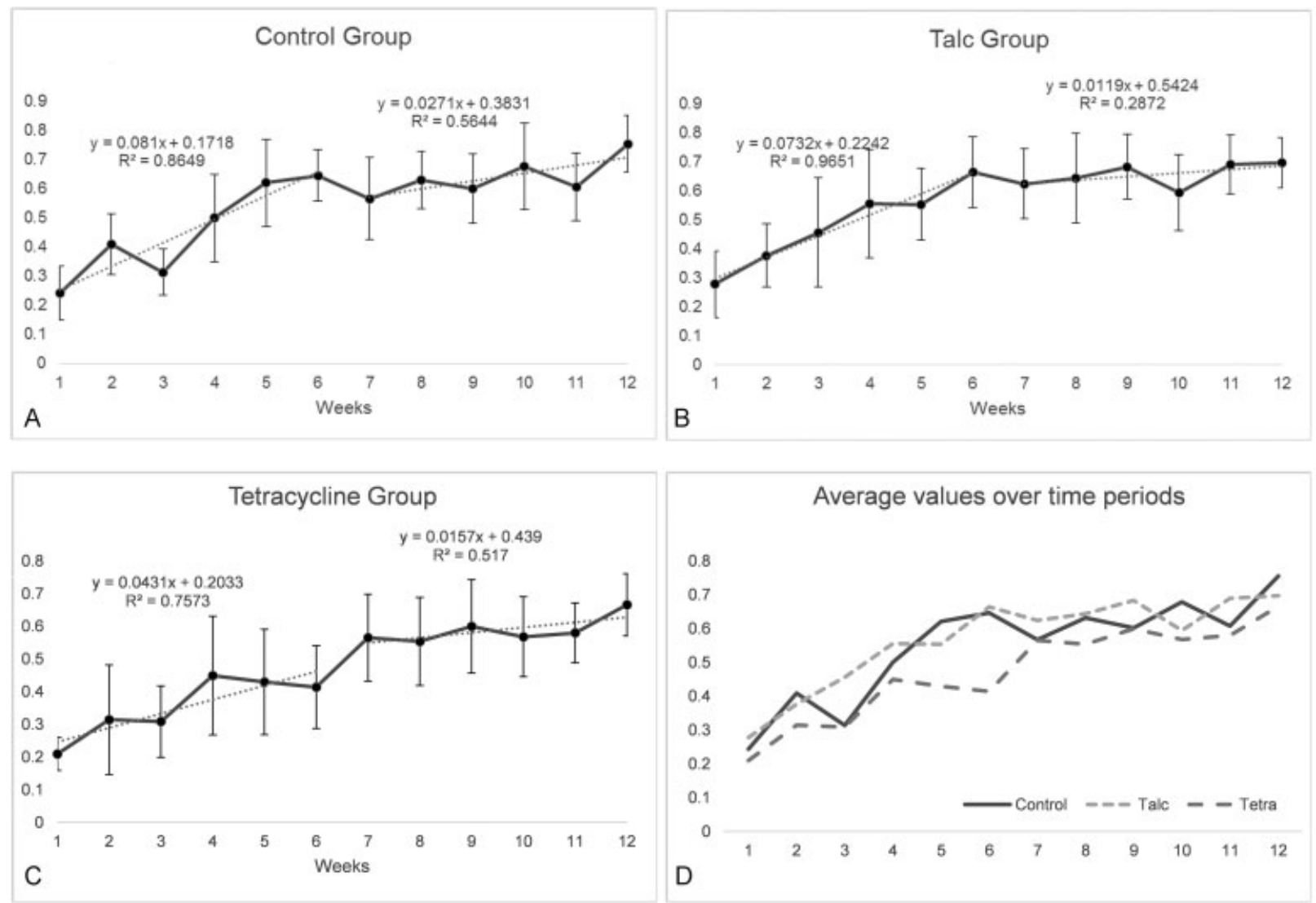

Fig. 1 Extensor postural thrust as percent recovery. The percent motor recovery for the (A) control group (B) talc group shows an increase in recovery (weeks, 1-6) followed by a plateau (weeks, 7-12). The recovery for the (C) tetracycline group is also similar, except for at the 6-week time point ( $p=0.007$ ) only at the 6 -week time point. The overall comparison of all three groups (D) over all time periods is similar, especially after week 6 . The total recovery for all three groups at 12 weeks were not significantly different.

The effect of adding potential scar inducing agents (e.g., talc and tetracycline) to the transected and repaired tibial nerve in the rat was also evaluated by histological staining at 12 weeks postoperatively. The normal appearance of the three sciatic nerve fascicles on the unoperated right side shows minimal connective tissue in the epineurium (mostly adipose tissue), small amounts of collagen, and blood vessels ( -Fig. 4A). At 12 weeks, the control group that received no additives following suture repair of the transected tibial nerve, the distal nerve contains substantial regeneration of small myelinated axons, however, the perineurium still lacked continuity (-Fig. 4B).

The appearance of regenerated axons in the endoneurium of the distal stump in the two groups with either tetracycline (-Fig. 5A) or talc (-Fig. 5B) was remarkably similar. At 12 weeks, the perineurium was not reconstituted in the distal stump and the epineurium of the latter two groups consistently demonstrated an infiltrate of inflammatory cells and debris that did not enter the endoneurial compartment containing axons. However, the presence of suture material in the endoneurial space in company with regenerated axons did provoke a chronic inflammatory response characterized by multinucleated giant cells ( - Fig. 6). The epineurium in this specimen from the tetracycline group had an abundance of collagen (blue staining). However, neither the giant cell response nor the abundance of collagen appeared to interfere with the nearby regenerated axons. This observed response was remarkably similar in all three repair groups, consistent with the group similarities for the PMR and muscle weight outcome measures.

\section{Discussion}

The results of this experimental study of potential nerve scaring agents (e.g., talc and tetracycline) suggest that in the rat tibial nerve model, regeneration of the transected axons across the lesion site into the distal stump and on to the target muscle was not impeded by these additives given at the time of surgery. Comparatively, human and rat nerve differ in their distribution on collagen. Humans have approximately a 5:1 ratio of epineurial to endoneurial hydroxyproline content, a surrogate marker for collagen. This ratio is much lower in rats at 1.9:1. We hypothesized the addition of extraneural sclerosing agents at the time of injury and recovery would increase the epineurial collagen content creating an environment for nerve healing more similar to human tissue. ${ }^{1}$ The suture line, despite the alteration of the barrier perineurium, permitted only limited infiltration of the scarring agent into the endoneurium. The same authors that described the collagen distribution also noted that as a result of injury, there is an increase in intraneural collagen downstream. ${ }^{1}$ Perhaps, our limited success in impeding recovery was a 


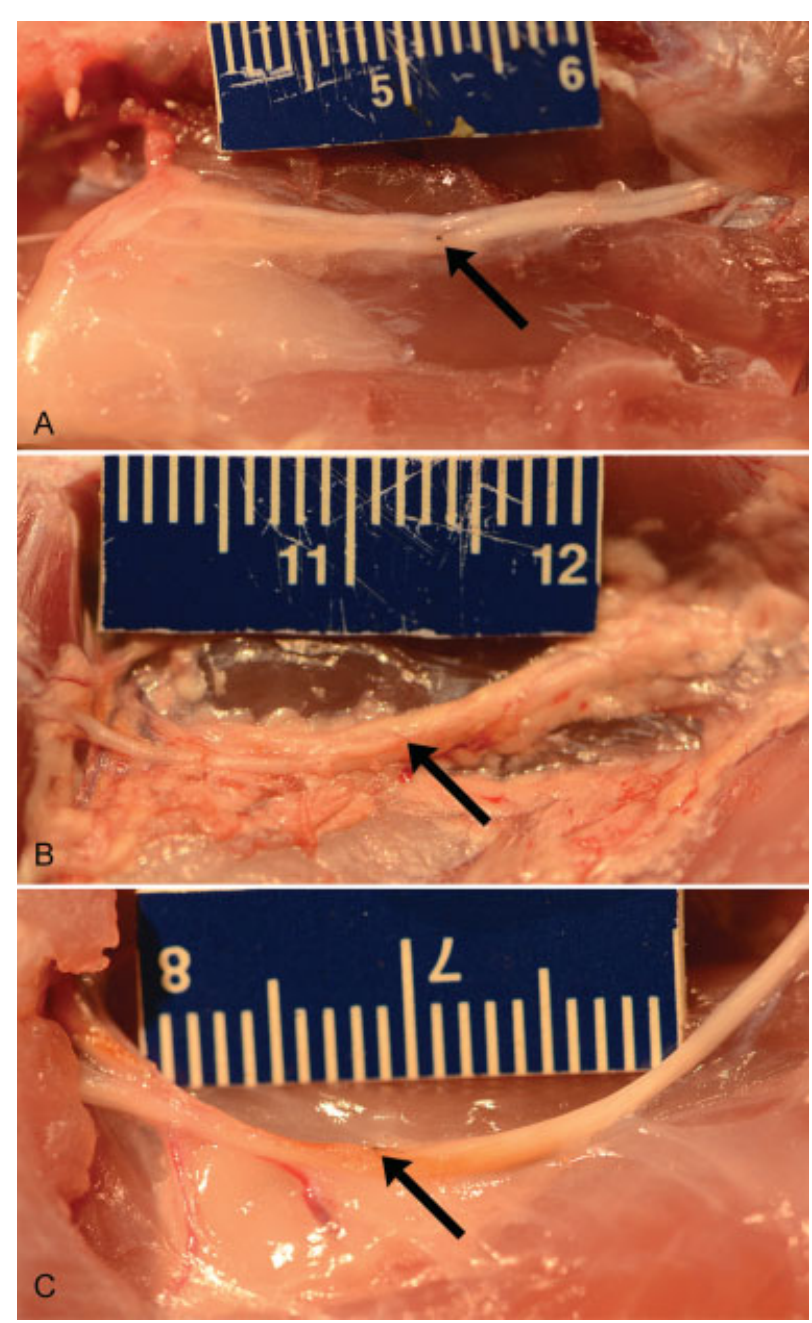

Fig. 2 The gross appearance of the left sciatic nerve from the (A) control group shows the tibial nerve suture site (arrow) and the distal fat pad in the left popliteal space. The (B) talc group has substantial debris remaining in the extraneural tissue space. The (C) tetracycline group displayed a yellow hue around the nerve.

result of only altering the environment at the site of injury. Our results are similar to previous research that attempted to alter the environment of the nerve by damaging the

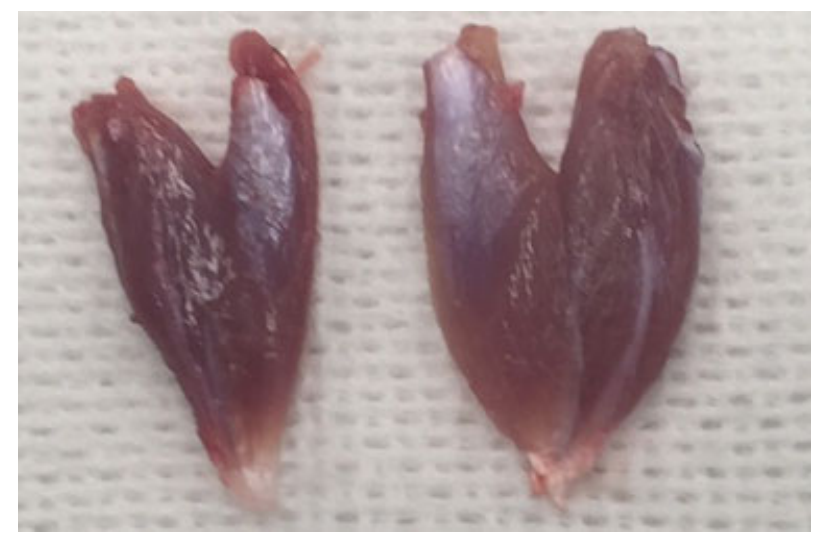

Fig. 3 The gross appearance of bilateral gastrocnemius muscles shows the extent of denervation atrophy in the control group. Left side denervated compared with right.

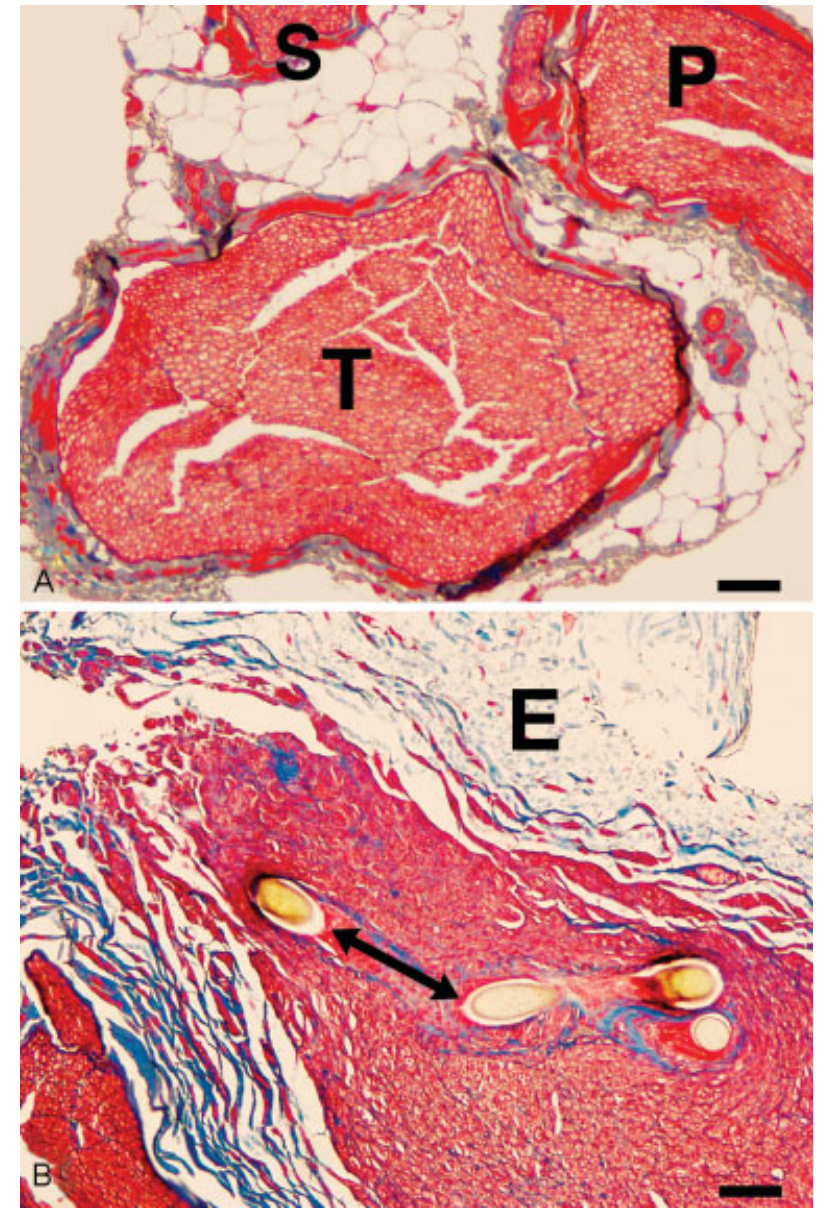

Fig. 4 At twelve weeks (A) the unoperated control nerve shows a tibial $(\mathrm{T})$ nerve fascicle accompanied by the peroneal $(\mathrm{P})$ and sural $(\mathrm{S})$ branches; the epineurium is adipose tissue and vessels. (B) The tibial nerve in the suture line (two-sided arrow) of the control group has regenerated myelinated axons into the distal stump. The perineurium has not fully reformed. The epineurium is denoted as (E). Masson's trichrome stain. Bar $=50$ microns.

surrounding tissue without directly injuring the epineurium, displaying the difficulty of using a rat model to exhibit functional characteristics of scar formation. ${ }^{11} \mathrm{Few}$ other studies exist that have attempted to increase the scar response with additive agents. However, it was shown in separate transgenic mice strains with mutations for increased or decreased inflammatory response and scarring, functional nerve recovery following transection predictably decreased and increased respectively. ${ }^{13}$ These findings were able to be duplicated with anti-inflammatory medications. ${ }^{5}$

The interval data from the EPT outcome were nearly identical for the three groups. The modest change at the 6week time-point affecting the positive slopes suggested the tetracycline group lagged behind the talc and control groups; the tetracycline group means at every time-point were the lowest of the three groups. The last 6 weeks of recovery resembled a plateau period with little additional recovery to final PMR values from $67 \%$ to $75 \%$, with no significant group differences. These comparisons were very similar to our previous results, ${ }^{12}$ comparing three different types of tibial 



Fig. 5 The regenerated tibial nerve at the suture line (arrows) is distinct from the debris and cellular infiltrate associated with the tetracycline (A) and talc (B) groups. Masson's trichrome stain. $\mathrm{Bar}=50$ microns.

nerve lesions using the tibial functional index (walking track analysis) over an 8-week postoperative period; the best recovery for a partial nerve lesion was $62 \%$. The PMR outcome measure tracks the recovery much like the sciatic (or tibial) functional index, but is easier to perform.

Similarly, the gastrocnemius wet weight ratios of the experimental side compared with the unoperated control side were nearly identical $(0.58-0.61)$. Typically, the denervation atrophy of muscle takes a longer time for recovery compared with function as demonstrated by the PMR. However, Brushart says the muscle weight ratio measure is not



Fig. 6 A transverse section of the tibial nerve from the tetracycline group at the suture line shows the multinucleated giant cell response (arrows) to three profiles of suture. Masson's tr ichrome stain. $\mathrm{Bar}=50$ microns. reliable. ${ }^{14}$ Correlations ranged from poor $(r=0.47$ at 12 weeks for the sciatic nerve) to moderate ( $r=0.70$ at 1 year for the tibial nerve). However, when we previously compared the correlation of muscle weight ratio to the tibial functional index for three different types of tibial nerve lesion at 9 weeks postoperative, the value was high $\left(r^{2}=0.895\right) .{ }^{15}$

Finally, histology at 12 weeks postoperative showed that regenerated axons in the distal stump were remarkably similar and unaffected by the epineurial presence of debris and inflammatory reactions. Most of the axons in the distal nerve segment were cut in cross section, indicating a longitudinal course for the regrowth. Quantitative analysis of the regenerated axons might confirm this impression, but the extra effort did not seem warranted. The 9-0 sutures are only 30 microns in diameter and coaptation at the time of repair only required $2-3$ sutures. Although the foreign body reaction to unresorbed suture material may have contributed to the incomplete recovery, it was similar in all three groups.

\section{Conclusion}

We conclude that the rat tibial nerve model does not resemble the situation found in human peripheral nerves from a clinical perspective. ${ }^{3,10}$ Perhaps another species with more epineurial tissue would respond to prospective agents administered. The rat exhibits a robust but still incomplete regeneration of damaged axons repaired by microsurgical methods following nerve transection. This was experimentally demonstrated long ago by Paul Weiss. ${ }^{16}$ The suture repair site was not significantly affected by either talc or tetracycline administered to the muscle bed of the nerves. We cannot rule out the possibility that these "scarring agents" may have inhibited vascular access to the nerve bed. Yet the majority of blood supply to a nerve is longitudinal and originates at the level of the limb joint. The tissues fixed by immersion had blood cells present in the lumina, suggesting good perfusion.

It remains to be demonstrated experimentally that specific agents will "scar" a nerve sufficiently to inhibit the progress of axonal regeneration and recovery. Other factors such as misdirected axonal growth and loss of specificity are probably the major contributors to the incomplete return of functional recovery. ${ }^{14}$ For example, crush lesions recover extremely well using a variety of outcome measures due to the fact that the basal lamina and Schwann cell tubes maintain their integrity following injury and throughout the recovery period. ${ }^{17-19}$ Although our histological methods were not designed to show such structural details, there did not appear to be widespread sprouting of axons into the epineurium like that which occurs during neuroma formation. An earlier study using the vibrating probe to detect growth cone currents at both the lesion site and distal regeneration front during the early periods following both nerve crush and transection provided such comparisons. ${ }^{8}$ Ngeow has reviewed the pertinent issues with respect to nerve scar formation and attempts to minimize and/or treat it, but the results are mixed. ${ }^{6}$ In conclusion, attempts to treat a scarred peripheral nerve as a clinical application will have to await the improvement of a potential scar model. 


\section{References}

1 Eather TF, Pollock M, Myers DB. Proximal and distal changes in collagen content of peripheral nerve that follow transection and crush lesions. Exp Neurol 1986;92(2):299-310

2 Ohsumi H, Hirata H, Nagakura T, et al. Enhancement of perineurial repair and inhibition of nerve adhesion by viscous injectable pure alginate sol. Plast Reconstr Surg 2005;116(3):823-830

3 Tetik C, Ozer K, Ayhan S, Siemionow K, Browne E, Siemionow M. Conventional versus epineural sleeve neurorrhaphy technique: functional and histomorphometric analysis. Ann Plast Surg 2002; 49(4):397-403

4 Görgülü A, Uzal C, Doğanay L, Imer M, Eliuz K, Cobanoğlu S. The effect of low-dose external beam radiation on extraneural scarring after peripheral nerve surgery in rats. Neurosurgery 2003;53(6): 1389-1395, discussion 1395-1396

5 Ozgenel GY. Effects of hyaluronic acid on peripheral nerve scarring and regeneration in rats. Microsurgery 2003;23(6):575-581

6 Ngeow WC. Scar less: a review of methods of scar reduction at sites of peripheral nerve repair. Oral Surg Oral Med Oral Pathol Oral Radiol Endod 2010;109(3):357-366

7 Park JS, Lee JH, Han CS, Chung DW, Kim GY. Effect of hyaluronic acidcarboxymethylcellulose solution on perineural scar formation after sciatic nerve repair in rats. Clin Orthop Surg 2011;3(4):315-324

8 Kerns JM, Fakhouri AJ, Weinrib HP, Freeman JA. Electrical stimulation of nerve regeneration in the rat: the early effects evaluated by a vibrating probe and electron microscopy. Neuroscience 1991; 40(1):93-107

9 Bilaceroglu S, Guo Y, Hawthorne ML, et al. Oral forms of tetracycline and doxycycline are effective in producing pleurodesis. Chest 2005;128(5):3750-3756
10 Watson J, Gonzalez M, Romero A, Kerns J. Neuromas of the hand and upper extremity. J Hand Surg Am 2010;35(3): 499-510

11 Zanjani L, Firouzi M, Nabian M-H, Nategh M, Rahimi-Movaghar V, S. Kamrani R. Comparison and evaluation of current animal models for perineural scar formation in rat. Iran J Basic Med Sci 2013;16(7):886-890

12 Hulata D, Hughes WF, Shott S, Kroin JS, Gonzalez MH, Kerns JM. Early behavioral and histological outcomes following a novel traumatic partial nerve lesion. J Neurosci Methods 2008;172(2): 236-244

13 Atkins S, Smith KG, Loescher AR, et al. Scarring impedes regeneration at sites of peripheral nerve repair. Neuroreport 2006;17; (12):1245-1249

14 Brushart TM. Nerve Repair. New York, NY: Oxford University Press; 2011

15 Malushte TS, Kerns JM, Huang CC, Shott S, Safanda J, Gonzalez M. Assessment of recovery following a novel partial nerve lesion in a rat model. Muscle Nerve 2004;30(5):609-617

16 Weiss P, Taylor AC. Histomechanical analysis of nerve reunion in the rat after tubular spicing. Arch Surg 1943;47(5):419-447

17 Kerns JM, Fakhouri AJ, Pavkovic IM. A twitch tension method to assess motor nerve function in rat. J Neurosci Methods 1987; 19(4):259-266

18 Kerns JM, Braverman B, Mathew A, Lucchinetti C, Ivankovich AD. A comparison of cryoprobe and crush lesions in the rat sciatic nerve. Pain 1991;47(1):31-39

19 Romano VM, Blair SJ, Kerns JM, Wurster RD. Comparison of fibrin glue, bioresorbable tubing and sutures in peripheral nerve repair. Restor Neurol Neurosci 1991;3(2):75-80 